\title{
Erratum to: Association study of H2AFZ with schizophrenia in a Japanese case-control sample
}

Daisuke Jitoku $^{1} \cdot$ Naoki Yamamoto ${ }^{1}$ - Yoshimi Iwayama ${ }^{2} \cdot$ Tomoko Toyota $^{2}$.

Momo Miyagi $^{1}$ - Takeshi Enokida ${ }^{1}$ Yuri Tasaka ${ }^{1}$ Masakazu Umino ${ }^{1}$.

Asami Umino ${ }^{1}$ - Akihito Uezato ${ }^{1}$ - Yasuhide Iwata ${ }^{3}$ - Katsuaki Suzuki ${ }^{3}$.

Mitsuru Kikuchi $^{4}$. Tasuku Hashimoto ${ }^{5}$. Nobuhisa Kanahara ${ }^{5} \cdot$ Akeo Kurumaji $^{1}$.

Takeo Yoshikawa $^{1,2} \cdot$ Toru Nishikawa $^{1}$

Published online: 11 August 2015

(C) Springer-Verlag Wien 2015

Erratum to: J Neural Transm (2015) 122:915-923

DOI 10.1007/s00702-014-1332-x

Table 1 contains several errors as originally published. Table 1 should read as follows; the corrected values are shown in italics.

The online version of the original article can be found under doi:10.1007/s00702-014-1332-x.

Toru Nishikawa

tnis.psyc@tmd.ac.jp

1 Department of Psychiatry and Behavioral Sciences, Tokyo Medical and Dental University Graduate School,

Tokyo 113-0034, Japan

2 Laboratory for Molecular Psychiatry, RIKEN Brain Science Institute, Wako, Saitama, Japan

3 Department of Psychiatry and Neurology, Hamamatsu University School of Medicine, Shizuoka, Japan

4 Department of Psychiatry and Neurobiology, Kanazawa University Graduate School of Medicine, Kanazawa, Japan

5 Department of Psychiatry, Graduate School of Medicine, Chiba University, Chiba, Japan 
Table 1 Allelic and genotypic analysis of the $H 2 A F Z$ gene

\begin{tabular}{|c|c|c|c|c|c|c|c|c|c|c|c|c|}
\hline \multirow[t]{2}{*}{ SNP ID rs number } & \multirow[t]{2}{*}{ Affection } & \multirow[t]{2}{*}{$N$} & \multirow[t]{2}{*}{ HWE $P$} & \multicolumn{2}{|c|}{ Allelic count } & \multirow[t]{2}{*}{ MAF } & \multirow[t]{2}{*}{ Allelic $P$} & \multirow[t]{2}{*}{ OR $(95 \%$ CI $)$} & \multicolumn{3}{|c|}{ Genotypic count } & \multirow[t]{2}{*}{ Genotypic $P$} \\
\hline & & & & $\mathrm{C}$ & $\mathrm{T}$ & & & & $\mathrm{CC}$ & $\mathrm{CT}$ & $\mathrm{TT}$ & \\
\hline SNP01 & $\mathrm{SCZ}$ & 1,999 & 0.001 & 3,995 & 3 & 0.001 & 1.000 & 1.081 & 1,997 & 1 & 1 & 0.486 \\
\hline \multirow[t]{2}{*}{ rs4594733 } & $\mathrm{CON}$ & 2,160 & 1.000 & 4,317 & 3 & 0.001 & $(1.000)$ & $(0.218-5.357)$ & 2,157 & 3 & 0 & $(0.886)$ \\
\hline & & & & A & $\mathrm{C}$ & & & & AA & $\mathrm{AC}$ & $\mathrm{CC}$ & \\
\hline SNP02 & SCZ & 2,008 & 0.033 & 3,048 & 968 & 0.241 & 0.114 & 1.086 & 1,139 & 770 & 99 & 0.236 \\
\hline \multirow[t]{2}{*}{ rs2276939 } & $\mathrm{CON}$ & 2,163 & 0.158 & 3,347 & 979 & 0.226 & $(0.377)$ & $(0.981-1.202)$ & 1,283 & 781 & 99 & $(0.567)$ \\
\hline & & & & $\mathrm{C}$ & $\mathrm{T}$ & & & & $\mathrm{CC}$ & $\mathrm{CT}$ & TT & \\
\hline SNP03 & SCZ & 1,983 & 0.264 & 2,864 & 1,102 & 0.278 & 0.640 & 1.024 & 1,044 & 776 & 163 & 0.421 \\
\hline \multirow[t]{2}{*}{ rs10014424 } & $\mathrm{CON}$ & 2,155 & 0.551 & 3,133 & 1,177 & 0.273 & $(0.942)$ & $(0.93-1.128)$ & 1,133 & 867 & 155 & $(0.839)$ \\
\hline & & & & A & G & & & & AA & AG & GG & \\
\hline SNP04 & SCZ & 1,986 & 0.964 & 1,906 & 2,066 & 0.480 & 0.058 & 0.9191 & 458 & 990 & 538 & 0.107 \\
\hline rs6854536 & $\mathrm{CON}$ & 2,147 & 0.244 & 2,151 & 2,143 & 0.501 & $(0.188)$ & $(0.8,431-1.002)$ & 525 & 1,101 & 521 & $(0.325)$ \\
\hline
\end{tabular}

Bold $P$ values indicate $P<0.05$

$N$ number of subjects, $H W E$ Hardy-Weinberg equilibrium, $M A F$ minor allele frequency, $O R$ odds ratio, $95 \% C I 95 \%$ confidence interval, $S C Z$ schizophrenia, CON control 